\title{
GRAIN GROWTH IN DYNAMICALLY RECRYSTALLIZED COPPER DURING ANNEALING ABOVE AND BELOW THE TEMPERATURE OF THERMALLY ACTIVATED NUCLEATION
}

\author{
M. V. Degtyarev, T. I. Chashchukhina, L. M. Voronova* \\ M.N. Miheev Institute of Metal Physics, Ural Branch, Russian Academy of Sciences, 18 S. Kovalevskoy St., \\ Ekaterinburg, Russian Federation \\ * Corresponding author e-mail: highpress@imp.uran.ru; address for correspondence: ul. S. Kovalevskoi 18, \\ Ekaterinburg, 620990 Russia. Tel.: +7 (343) 3783818
}

The effect of dynamic recrystallization on the change of the structure of copper (99.97\%), deformed by the "shear under pressure" and ECAP methods, during subsequent heating is studied. It is shown that different conditions of deformation of copper provide varying degrees of dynamic recrystallization, dynamic recovery and strain hardening. A submicrograin structure fails to be formed after primary recrystallization is completed. Dynamic recrystallization leads to the formation of the coarsest grain $(15-20 \mu \mathrm{m})$ and size heterogeneous structure during subsequent recrystallization at $100{ }^{\circ} \mathrm{C}$ (below the temperature of thermally activated nucleation in moderately deformed copper). Heating at temperatures ranging between $150{ }^{\circ} \mathrm{C}$ and $400{ }^{\circ} \mathrm{C}$ (above the temperature of thermally activated nucleation) leads to the formation of finer grains in the fully recrystallized material. In samples with a partially dynamically recrystallized structure the grain size changes insignificantly. The finest grain with an average size of $4-7 \mu \mathrm{m}$ is formed after short-time annealing at $300{ }^{\circ} \mathrm{C}$.

Keywords: severe plastic deformation, copper, temperature-compensated strain rate, dynamic recrystallization, static recrystallization, structure.

DOI: $10.17804 / 2410-9908.2016 .5 .015-029$

\section{References}

1. Gorelik S.S., Dobatkin S.V., Kaputkina L.M. Rekristallizatsiya metallov i splavov [Recrystallization of Metals and Alloys]. Moscow, MISIS Publ., 2005, 432 p. (In Russian).

2. Voronova L.M., Degtyarev M.V., Chashchukhina T.I. Recrystallization of the ultradispersed structure of pure iron formed at different stages of the deformation-induced strain hardening. Physics of Metals and Metallography, 2007, vol. 104, no. 3, pp. 262-273. DOI: 10.1134/S0031918X07090086.

3. Krasnoperova Yu.G., Degtyarev M.V., Voronova L.M., Chashchukhina T.I. Effect of Annealing Temperature on the Recrystallization of Nickel with Different Ultradisperse Structures. Physics of Metals and Metallography, 2016, vol. 117, no. 3, pp. 267-274. DOI: 10.1134/S0031918X16030078.

4. Smirnova N.A., Levit V.I., Pilyugin V.P., Kuznetsov R.I., Degtyarev M.V. Peculiarities of low-temperature recrystallization of nickel and copper. Fizika Metallov i Metallovedenie, 1986, vol. 62 , iss. 3 , pp. 566-570.

5. Degtyarev M.V., Chashchukhina T.I., Romanova M.Yu., Voronova L.M. Correlation between the copper structure and temperature-rate parameters of pressure-induced shear deformation. Doklady Physics, 2004, vol. 49, no. 7, pp. 415-418. DOI: 10.1134/1.1784855.

6. Degtyarev M.V., Chashchukhina T.I., Voronova L.M., Patselov A.M., Pilyugin V.P. Influence of the relaxation processes on the structure formation in pure metals and alloys under high-pressure deformation. Acta Materialia, 2007, vol. 55, pp. 6039-6050. DOI: 10.1016/j.actamat.2007.04.017.

7. Gindin I.A., Lazarev B.G., Starodub J.D., Lazareva M.B. Low-Temperature Recrystallization of Copper Rolled at 77 and 20 K. Dokl. Akad. Nauk SSSR, 1966, vol. 171, no. 3, pp. 552-554. 
8. Bykov V.M., Likhachev V.A., Nikonov Yu.A., Serbina L.L., Shibalova L.I. Fragmentation and dynamic recrystallization of copper at large and very large plastic deformations. Fizika Metallov i Metallovedenie, 1978, vol. 45, no. 1, pp. 163-169.

9. Chuvil'deev V.N., Kopylov V.I., Nokhrin A.V., Makarov I.M., Malashenko L.M., Kukareko V.A. Anomalous grain growth in nano- and microcrystalline metals produced by equal-channel angular pressing methods. Part I. Structural studies. Materialovedenie, 2003, no. 4, pp. 9-17.

10. Kopylov V.I., Makarov I.M., Nesterova E.V., Rybin V.V. Crystallographic analysis of a submicrocrystalline structure obtained by ECA pressing of highly pure copper. Voprosy materialovedeniya, 2002, no. 1 (29), pp. 273-278. (In Russian).

11. Amirkhanov N.M., Islamgaliev R.K., Valiev R.Z. Thermal Relaxation and Grain Growth upon Isothermal Annealing of Ultrafine-Grained Copper Produced by Severe Plastic Deformation. Fizika Metallov i Metallovedenie, 1998, vol. 86, iss. 3, pp. 99-105.

12. Orlova D.K., Chashchukhina T.I., Voronova L.M., Degtyarev M.V., Krasnoperova Yu.G. Effect of impurities on dynamic recrystallization in copper deformed in bridgman anvils. Diagnostics, Resource and Mechanics of materials and structures, 2015, iss. 5, pp. 90-98. DOI: 10.17804/2410-9908.2015.5.090-098. Available at: http://dreamjournal.org/DREAM_Issue_5_2015_Orlova_D.K._et_al._090_098.pdf (accessed 15.09.2016).

13. Shirinkina I.G., Brodova I.G., Astafiev V.V. Thermal stability of the ultrafine AMTs aluminum alloy after high strain-rate deformation. Diagnostics, Resource and Mechanics of materials and structures, 2015, iss. 5, pp. 72-79. DOI: 10.17804/2410-9908.2015.5.072-079. Available at: http://dream-journal.org/DREAM_Issue_5_2015_Shirinkina_I.G._et_al._072_079.pdf (accessed 13.09.2016).

14. Gusev A.I. Nanokristallicheskie materialy: metody polucheniya i svoistva [Nanocrystalline Materials: Production Methods and Properties.]. Ekaterinburg, UrO RAN Publ., 1998, 200 p. (In Russian).

15. Pilyugin V.P. Structural and phase transformations in iron alloys under high pressure deformation. Ph.D thesis, Ekaterinburg, 1993. (In Russian).

16. Chashchukhina T.I., Degtyarev M.V., Voronova L.M. Effect of Pressure on the Evolution of Copper Microstructure upon Large Plastic Deformation. Physics of Metals and Metallography, 2010, vol. 109, iss. 2, pp. 201-209. DOI: 10.1134/S0031918X10020122.

17. Segal V.M., Reznikov V.I., Kopylov V.I., Pavlik L.A., Malyshev V.F. Protsessy plasticheskogo strukturoobrazovaniya metallov [Processes of Plastic Structure Formation of Metals]. Minsk, Navuka i tekhnika Publ., 1994, 232 p. (In Russian).

18. Degtyarev M.V., Chashchukhina T.I., Voronova L.M., Kopylov V.I. Establishment of conformity between the true strain, hardness and size of the structural elements of iron and structural steel under severe plastic deformation in different ways. Fizicheskaya mezomekhanika, 2013, vol. 16, no. 6, pp. 71-80. (In Russian).

19. Degtyarev M.V., Voronova L.M., Chashchukhina T.I. Low-temperature recrystallization of pure iron deformed by shear under pressure. Physics of Metals and Metallography, 2004, vol. 97, no. 1 , pp. $72-81$.

20. Frolova N.Yu., Zeldovich V.I., Khomskaya I.V., Kheifets A.E., Shorokhov E.V. Influence of aging and strain on the structure and mechanical properties of chromium-zirconium bronze. Diagnostics, Resource and Mechanics of materials and structure, 2015, iss. 5, pp. 99-108. DOI: 10.17804/2410-9908.2015.5.099-108. Available at: http://dreamjournal.org/DREAM_Issue_5_2015_Frolova_N._Yu._et_al._099_108.pdf (accessed 13.09.2016). 
Подана в журнал: 22.09 .2016

УДК 669.3:539.89

DOI: $10.17804 / 2410-9908.2016 .5 .015-029$

\title{
РОСТ ЗЕРНА В ДИНАМИЧЕСКИ РЕКРИСТАЛЛИЗОВАННОЙ МЕДИ ПРИ ОТЖИГЕ ВЫШЕ И НИЖЕ ТЕМПЕРАТУРЫ ТЕРМОАКТИВИРОВАННОГО ЗАРОЖДЕНИЯ
}

\author{
М. В. Дегтярев, Т. И. Чащухина, Л. М. Воронова*
}

\begin{abstract}
Федеральное государственное бюджетное учреждение науки Институт физики металлов имени М.Н. Михеева Уральского отделения Российской академии наук, ул. С. Ковалевской, 18, Екатеринбург, Российская Федерация
\end{abstract}

\begin{abstract}
*Ответственный автор. Электронная почта: highpress@imp.uran.ru; Адрес для переписки: ул. С.Ковалевской, 18, 620990, Екатеринбург, Российская Федерация. Телефон: +7 (343) 378-38-18
\end{abstract}

Исследовано влияние динамической рекристаллизации на изменение при последующем нагреве структуры меди марки М0б, деформированной методами «сдвиг под давлением» и РКУП. Показано, что разные условия деформации меди обеспечивают различную степень развития динамической рекристаллизации, динамического возврата и деформационного упрочнения. По завершении первичной рекристаллизации не удается получить субмикрозернистую структуру. Динамическая рекристаллизации приводит к формированию наиболее крупнозернистой (15-20 мкм) и размерно неоднородной структуры при последующей статической рекристаллизации, осуществляемой при $100{ }^{\circ} \mathrm{C}$ (ниже температуры термоактивированного зарождения в умеренно деформированной меди). Нагрев в интервале $150-400{ }^{\circ} \mathrm{C}$ (выше температуры термоактивированного зарождения) приводит к формированию более мелкого зерна в полностью динамически рекристаллизованном материале и мало меняет размер зерна в образцах с частично динамически рекристаллизованной структурой. Наиболее мелкое зерно средним размером 4-7 мкм формируется после кратковременного отжига при $300{ }^{\circ} \mathrm{C}$

Ключевые слова: большая пластическая деформачия, медь, температурноскомпенсированная скорость деформаџии, динамическая рекристаллизачия, статическая рекристаллизаџия, структура.

\section{1. Введение}

Условия пластической деформации (температура, скорость, степень деформации) оказывают существенное влияние на развитие как динамической рекристаллизации при деформации, так и статической рекристаллизации при последующем отжиге. В материалах, деформация которых не сопровождается динамической рекристаллизацией, закономерности рекристаллизации при последующем отжиге хорошо изучены [1-3]. В частности установлено, что при создании в результате большой пластической деформации однородной субмикрокристаллической (СМК) структуры изменяется кинетика первичной рекристаллизации, что обеспечивает высокую термическую стабильность такой структуры и сохранение высокого комплекса механических свойств, полученных в результате деформации [2]. Еще одно существенное следствие образования СМК структуры при большой пластической деформации снижение температуры начала рекристаллизации и возможность осуществления так называемой низкотемпературной рекристаллизации, протекающей ниже температуры термоактивированного зарождения в умеренно деформированном материале [4]. Исследования, проведенные на железе и никеле, показали, что в результате низкотемпературной рекристаллизации СМК структуры может сформироваться зерно более крупное, чем при более высокой температуре отжига. Измельчение происходит за счет подключения термоактивированного зарождения $[2,3]$. Снижение температуры рекристаллизации после большой пластической 
деформации принципиально меняет подход к изучению материалов с низкой гомологической температурой, например меди [5]. Большой деформации меди из-за высокой пластичности посвящено много исследований [6-11]. Обычно эволюцию структуры меди описывают в терминах холодной деформации, когда структура формируется в условиях непрерывного накопления дефектов [9-11]. Если подходить к большой пластической деформации меди, как к горячей деформации, то формирующаяся структура будет определяться не только величиной деформации (e), но и температурно-скомпенсированной скоростью деформации $(\ln Z)$ [12]. Этот параметр определяет соотношение процессов накопления дефектов, динамического возврата и динамической рекристаллизации. Ранее на меди с разным содержанием примесей были установлены значения $e$ и $\ln Z$, при которых в структуре в ходе деформации сдвигом под давлением появляются первые центры рекристаллизации $(e \approx 2, \ln Z \leq 42)$. Также были определены условия, при которых формируется полностью или частично рекристаллизованная структура ( $35<\ln Z<38$ и $38<\ln Z<42$ соответственно). Образование при динамической рекристаллизации неоднородной структуры позволяет объяснить, почему процесс последующей статической рекристаллизации в меди носит «необычный аномальный» характер, связанный с существенным укрупнением только отдельных зерен $[9-11,13]$. Отсутствие закономерностей в развитии рекристаллизации материалов с низкой гомологической температурой не позволяет прогнозировать поведение таких материалов и изменение их свойств при эксплуатации, что обусловливает необходимость исследования термической стабильности таких материалов.

Наиболее распространенными методами получения структуры субмикронного масштаба являются сдвиг под давлением и равноканальное угловое прессование (РКУП) [14]. Считается, что в первом случае активный теплоперенос через массивные наковальни препятствует разогреву образца [15], а при РКУП возможен разогрев до $120{ }^{\circ} \mathrm{C}$ [10]. Поэтому в формировании структуры при РКУП существенная роль принадлежит динамическому возврату. Напротив, приложение значительно более высокого, чем при РКУП, внешнего давления в наковальнях Бриджмена затрудняет развитие динамического возврата [16].

Для изучения влияния структурообразующих процессов при деформации на кинетику статической рекристаллизации были выбраны режимы деформации меди, обеспечивающие разную степень развития динамической рекристаллизации: появление первых динамически рекристаллизованных зерен (сдвиг под давлением с углом поворота наковальни $45^{\circ}$ ), полную динамическую рекристаллизацию структуры (сдвиг под давлением, 5 оборотов наковальни) и сочетание динамической рекристаллизации и динамического возврата (РКУП).

\section{2. Материал и методика}

Медь марки М0б (чистотой 99,97 \%) для получения ультрадисперсной зеренносубзеренной структуры деформировали РКУП по режиму «Б» с углом пересечения каналов $90^{\circ}$ [17] и числом циклов $\mathrm{N}=16$. Часть деформированного материала рекристаллизовали при $873 К, 1 ч$. После отжига средний размер рекристаллизованного зерна составляет 60 мкм. В дальнейшем отожженные образцы диаметром 5 мм, толщиной 0,3 мм подвергли деформации сдвигом под давлением 6 ГПа с углом поворота наковальни $45^{\circ}$ и 5 оборотов. Образцы, деформированные обоими методами, отжигали при $100{ }^{\circ} \mathrm{C}$ от 1 до 10 ч, при $150{ }^{\circ} \mathrm{C}, 200{ }^{\circ} \mathrm{C}$, $300{ }^{\circ} \mathrm{C}$ и $400{ }^{\circ} \mathrm{C}$ от 3 мин до 1 ч.

Методика расчета истинной деформации при сдвиге под давлением и РКУП обоснована в статье [18]. Скорость деформации при сдвиге составляет $10^{-1}>\dot{e}>10^{-4} \mathrm{c}^{-1}$, при РКУП $\dot{e} \sim 1$ $\mathrm{c}^{-1}$. В настоящей работе истинная деформация при РКУП составляет $e=3,5$. При РКУП происходит неконтролируемый нагрев образца и температура деформации может изменяться от 20 до $120{ }^{\circ} \mathrm{C}$. То есть при РКУП значения $\ln Z$ могут находиться в интервале от 43 до 33 . Истинная деформация при сдвиге под давлением зависит от расстояния от центра образца (r) [18]. На середине радиуса образца $\mathrm{r} \approx 1,5$ мм параметры деформации составили: для угла по- 
ворота наковальни $45^{\circ}-e=3,5, \ln Z=42$, для 5 полных оборотов наковальни $-e=9,1 \ln Z=38,3$. При сдвиге под давлением экспериментальная погрешность определения величины деформации не превосходила $\pm 0,2$ единицы истинной деформации, а температурноскомпенсированной скорости деформации $\Delta \ln Z= \pm 0,5$.

Микротвердость после деформации измеряли на приборе ПМТ-3 при нагрузке 0,125H на полированных шлифах. Нагрузку выбирали исходя из толщины образцов: для корректных измерений толщина образца должна превышать диагональ отпечатка, по крайней мере в 1,5 раза. После сдвига под давлением измерения проводили на расстоянии $1,5 \pm 0,5$ мм от центра образца. Для каждого состояния было проведено не менее 10 измерений микротвердости, коэффициент вариации значений составил около $3 \%$, что соответствует погрешности $0,03-$ 0,05 ГПа.

Структуру меди после деформации и отжига исследовали методами просвечивающей и сканирующей электронной микроскопии (ТЕМ и СЭМ соответственно) на приборах JEM 200CX и QUANTA-200 Philips, а также с помощью оптического микроскопа (OM) NEOPHOT-2 на травленых шлифах. После сдвига под давлением структуру исследовали на

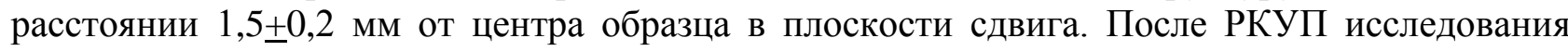
проводили в плоскости, перпендикулярной направлению последнего сдвига. Размеры рекристаллизованных зерен определяли методом секущих по СЭМ и ОМ изображениям, а размеры микрокристаллитов и зерен менее 5 мкм прямыми измерениями по ПЭМ изображениям. Строили гистограммы распределения элементов структуры по размерам, определяли средний размер $\left(d_{\mathrm{cp}}\right)$ и среднеквадратичное отклонение $(\sigma)$, которое использовали для оценки погрешности. Долю рекристаллизованной структуры определяли по ПЭМ и СЭМ изображениям как долю площади, занятой рекристаллизованными зернами.

\section{3. Результаты и их обсуждение}

На рис. 1 приведена структура, сформированная в меди после деформации РКУП и сдвигом под давлением. После РКУП и поворота наковальни на $45^{\circ}$ достигается одинаковая расчетная истинная деформация $(e=3,5)$, однако благодаря разным условиям деформации существуют различия в структуре и микротвердости. После РКУП микротвердость меди в 1,5 раза превышает микротвердость крупнокристаллического материала, но это упрочнение слабее, чем в результате деформации сдвигом под давлением: 1,10 и 1,25 ГПа соответственно. Видно (рис. $1 a, \sigma)$, что деформация РКУП не привела к образованию однородной по типу структуры, в ней присутствуют дислокационные ячейки, субзерна и рекристаллизованные зерна, средний размер элементов структуры составляет 0,4 мкм. Структура, созданная при сдвиге под давлением с $e=3,5$, также неоднородна и содержит такие же составляющие (рис. 1 $8,2)$. Средний размер элементов структуры меньше - 0,3 мкм. Можно сказать, что гистограммы распределения элементов структуры по размерам после РКУП (рис. 2 a) и после сдвига под давлением с $e=3,5$ (рис. 2 б) похожи: обе гистограммы имеют второй небольшой максимум в области размеров свыше 1,8 мкм (рис. 2 a) и 1,5-1,8 мкм (рис. 2 б) соответственно. Однако эти максимумы образованы разными структурными составляющими: после РКУП это конгломераты субзерен близкой ориентировки и дислокационные ячейки (рис. 1 б), а после сдвига это рекристаллизованные зерна (рис. 1 в). Ранее было показано [6, 16], что в меди после окончания деформации проходит постдинамическая рекристаллизация (ПДР), т.е. зерна размером более 1 мкм, наблюдающиеся в структуре после сдвига под давлением, вероятнее всего результат ПДР и следовательно имеют пониженную дефектность. Согласно температурно-скоростным условиям деформации $(\ln Z=42)$ структура меди при сдвиге под давлением на $45^{\circ}$ формируется на стадии частичной ДР, причем доля ДР невелика, т.е. невелико число центров ПДР, а движущая сила ПДР, напротив, высокая. Несмотря на это твердость после РКУП ниже, чем после сдвига под давлением, что может косвенно свидетельствовать о более глубоком протекании возврата в первом случае. 

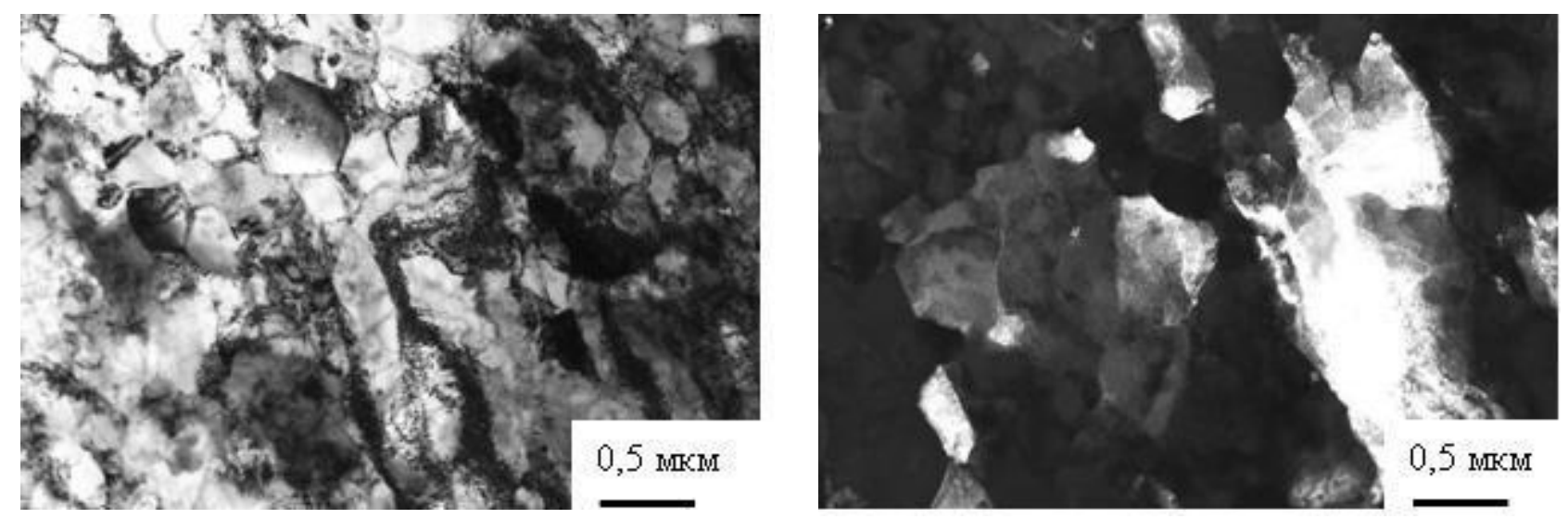

$a$

6
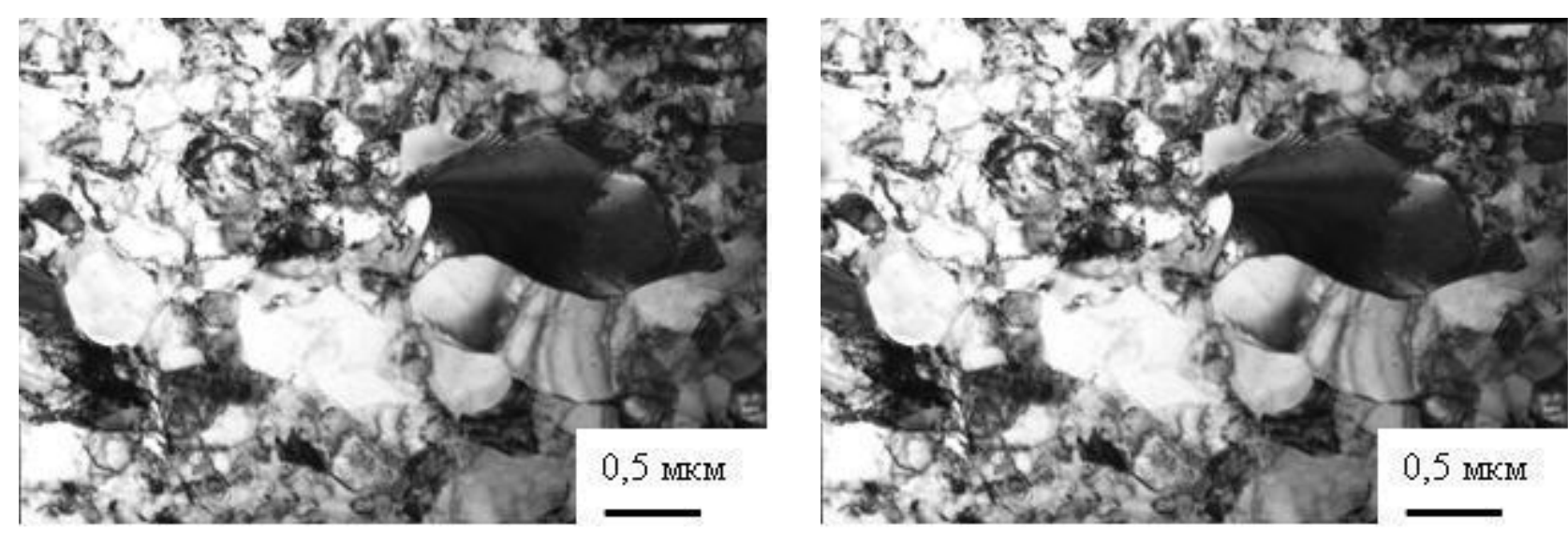

B

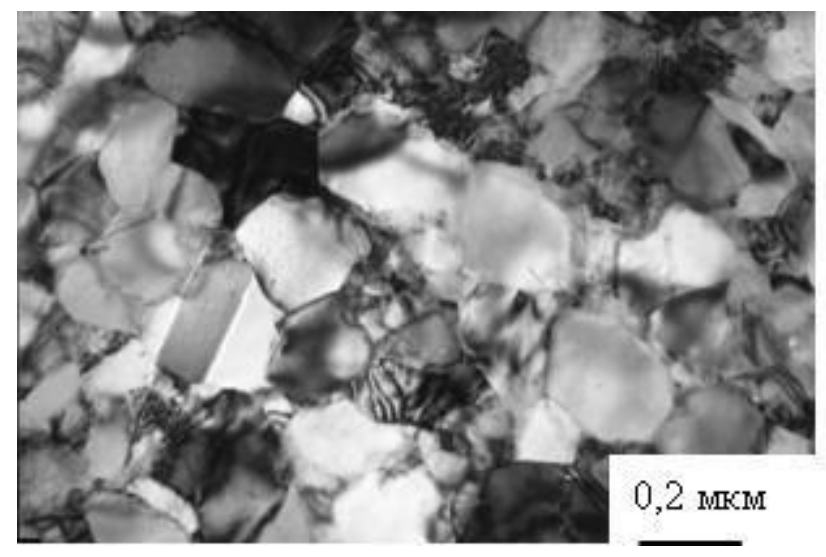

$\partial$

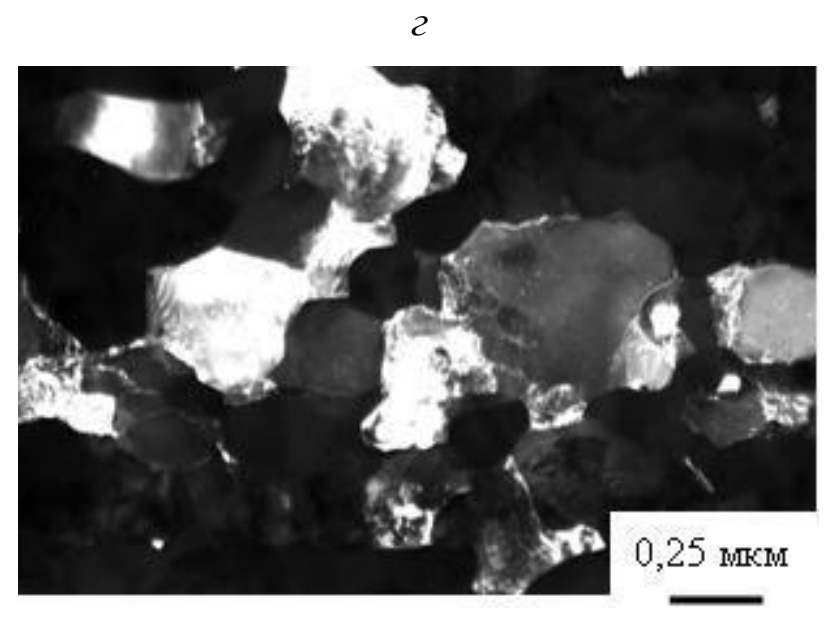

$e$

Рис. 1. Микроструктура меди после деформации РКУП $(a, \sigma)$ и сдвигом под давлением с поворотом наковальни на $45^{\circ}(6,2)$ и 5 оборотов $(\partial, e)$ 
open-aCCeSS journal

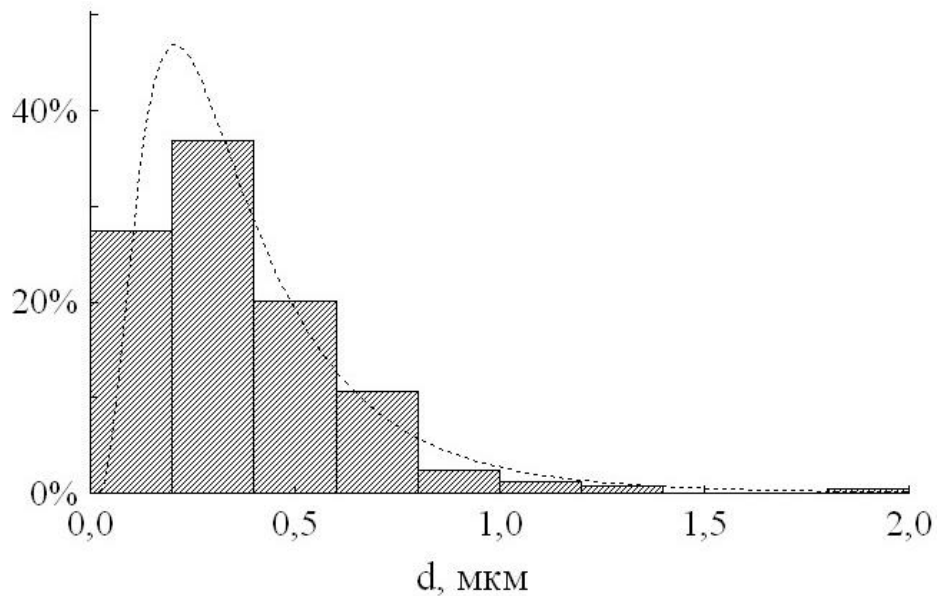

$a$

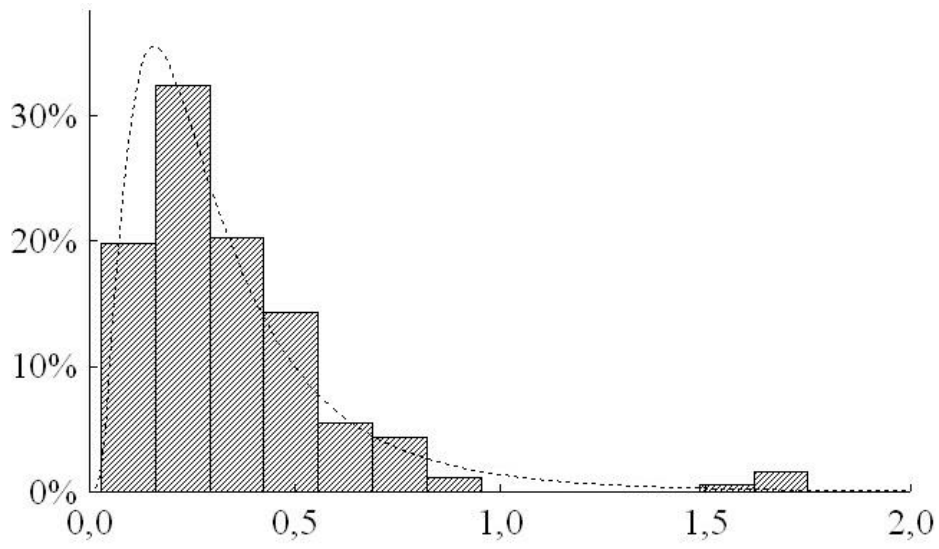

d, MKM

$\sigma$

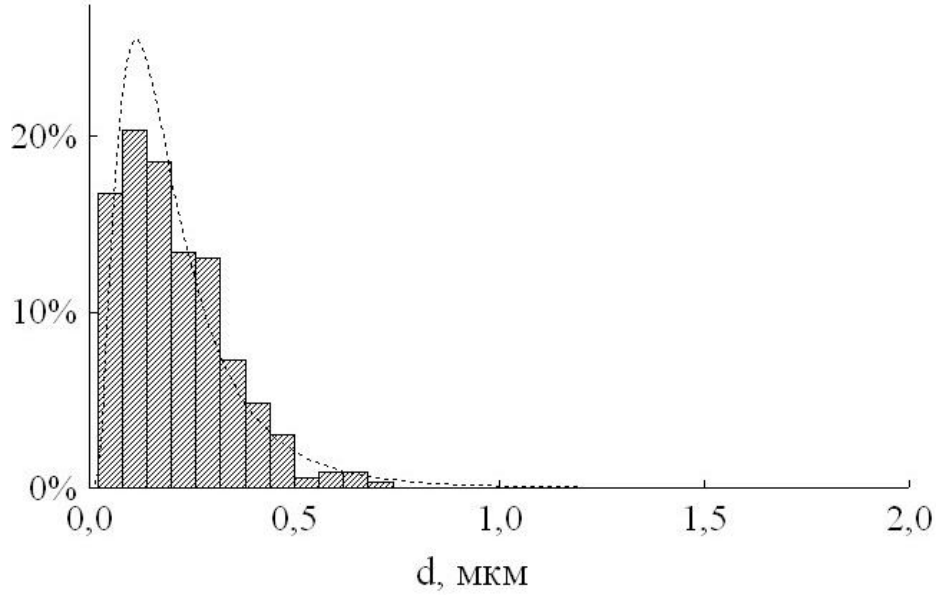

B

Рис. 2. Распределение элементов структуры по размерам после деформации меди РКУП (a) и сдвигом под давлением с поворотом наковальни на $45^{\circ}$ (б) и 5 оборотов (в)

Деформация на 5 оборотов наковальни завершается на стадии полной ДР, и в структуре мы наблюдаем рекристаллизованные зерна (рис. $1 \partial$ ). Некоторые из них содержат субструктуру (рис. 1 e), что связано с чередованием рекристаллизации и наклепа при деформации на стадии ДР. Интересно, что рекристаллизованные зерна часто имеют близкую ориентировку, что говорит об аксиальной текстуре. Присутствие двойников отжига в некоторых зернах свидетельствует о ПДР, но движущая сила роста зерна невелика. Гистограмма распределения зерен по размером достаточно узкая, максимальный размер менее 1 мкм 
(рис. 2 в). Меньший размер зерна $\left(d_{\mathrm{cp}}=0,2\right.$ мкм) обусловливает большую микротвердость $(\mathrm{H}=1,55$ ГПа).

В настоящей работе провели отжиги меди при температурах 100, 150, 200, 300 и $400{ }^{\circ} \mathrm{C}$ разной длительности. Следует отметить, что согласно данным работы [10] температура рекристаллизации меди М0б составляет $120^{\circ} \mathrm{C}$. Таким образом, статическая рекристаллизация при более низкой температуре возможна только из центров, созданных при деформации (низкотемпературная рекристаллизация), а при отжиге выше этой температуры возможно образование зародышей рекристаллизации термоактивируемым путем, т.е. к центрам, сформированным при деформации, добавятся новые центры рекристаллизации. Следовательно, отжиг при 100 и $150{ }^{\circ} \mathrm{C}$ проведен при температурах ниже и вблизи термоактивированного зарождения, а отжиг при $200-400^{\circ} \mathrm{C}$ - выше температуры термоактивированного зарождения.

Размер рекристаллизованного зерна определяется соотношением скорости формирования центров и скорости их роста [1]. Измельчить зерно можно, повысив плотность зародышей, способных к росту (увеличением степени деформации и повышением температуры нагрева) и снизив скорость их роста (понижением температуры). Большая пластическая деформация позволяет повысить плотность центров за счет суммирования зародышей, сформировавшихся при деформации и термически активируемых при нагреве. При исследовании чистого железа было показано, что при низкотемпературной рекристаллизации субмикрокристаллической структуры формируется термически стабильная субмикрозернистая структура сотового типа, благодаря большой плотности микрокристаллитов и низкой скорости их роста [19].

Центры рекристаллизации до нагрева могут быть созданы за счет ДР - это «чистые» зерна, сформировавшиеся перед окончанием деформации и поэтому имеющие наименьшую дефектность. Плотность центров наибольшая в области полной ДР (5 оборотов), при этом окружающие их зерна имеют разную степень наклепа. Это определяет различную движущую силу роста в разных направлениях. На стадии отдельных ДР зерен (45 градусов) центры рекристаллизации окружены деформированной матрицей с ячеистой структурой и движение границ в разных направлениях равновероятно. Структурные исследования показывают, что после РКУП плотность центров рекристаллизации такая же, как на стадии отдельных ДР зерен, но окружающая матрица имеет преимущественно субзеренную структуру, стабильную при нагреве и в отличие от ячеистой структуры не обеспечивающую градиент плотности дислокаций через высокоугловую движущуюся границу.

На рисунках 3 и 4 приведены кинетические зависимости изменения размера зерна от времени выдержки при исследованных температурах.
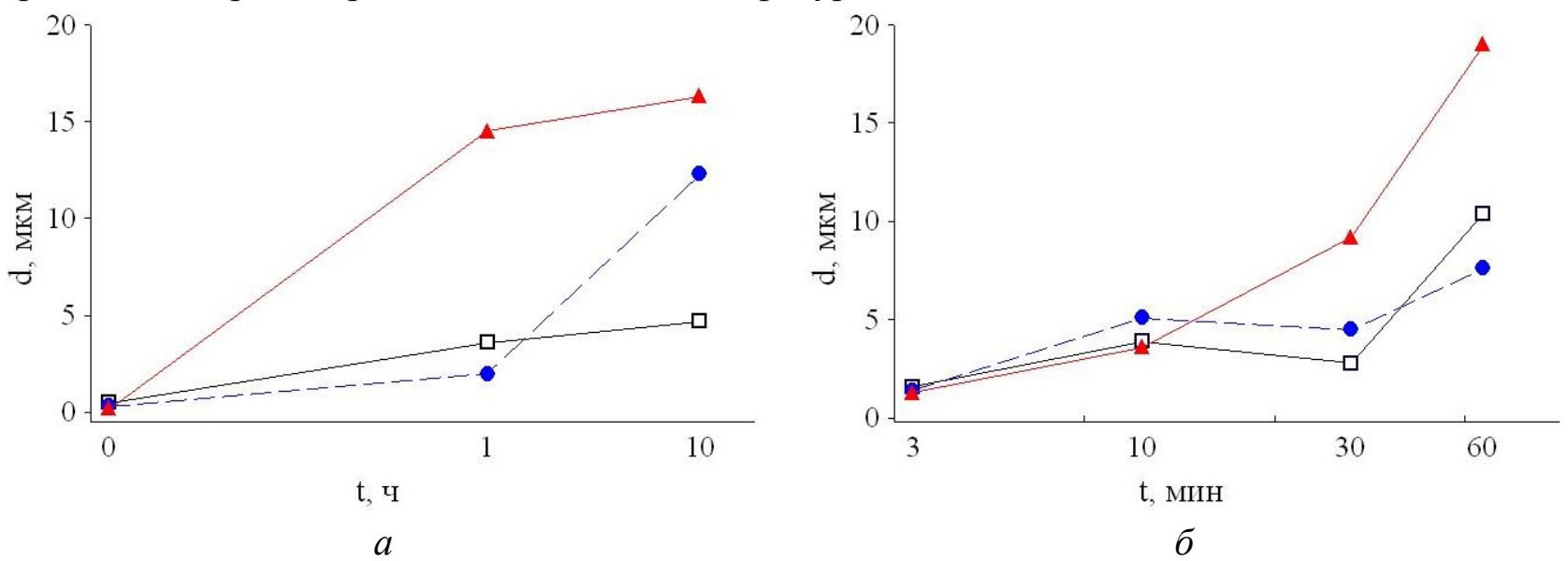

6

Рис. 3. Кинетические зависимости изменения размера зерна от времени выдержки при $100(a)$ и $150{ }^{\circ} \mathrm{C}(б)$ после деформации РКУП () и сдвигом под давлением с поворотом наковальни на $45^{\circ}(\bullet)$ и 5 оборотов $(\boldsymbol{\Delta})$ 
open-acCess journal

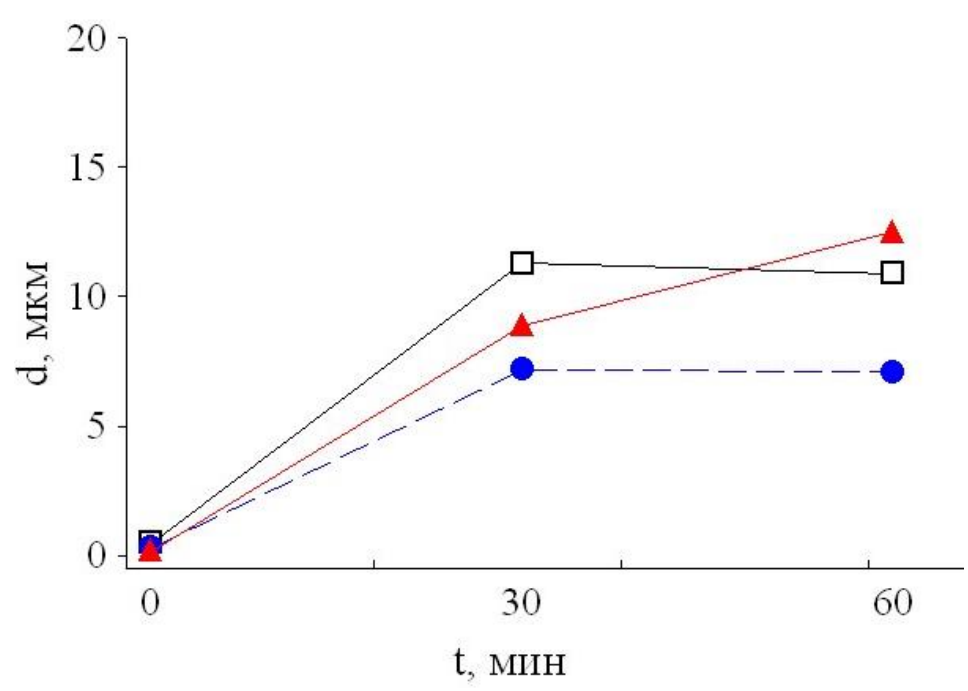

$a$
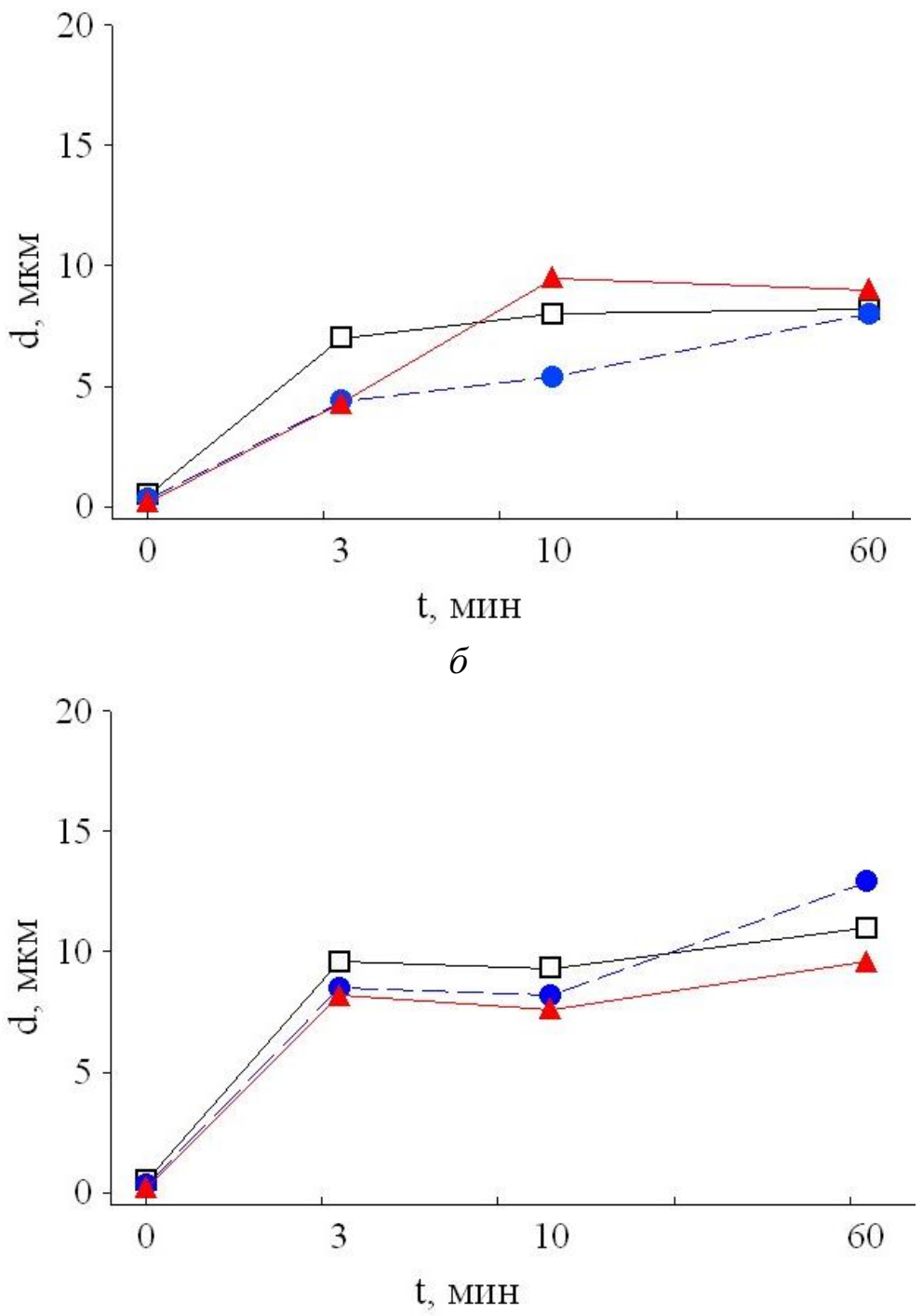

B

Рис. 4. Кинетические зависимости изменения размера зерна от времени выдержки при $200(a), 300$ (б) и $400{ }^{\circ} \mathrm{C}$ (в) после деформации РКУП () и сдвигом под давлением с поворотом наковальни на $45^{\circ}(\bullet)$ и 5 оборотов $(\boldsymbol{\Delta})$

Degtyarev M.V. et al. / Grain growth in dynamically recrystallized copper during annealing above and below the temperature of thermally activated nucleation 
При всех температурах нагрева независимо от степени протекания ДР в исследованной меди не сохраняется субмикронный размер зерна. Даже в медных сплавах, несмотря на выделение дисперсных частиц, не удается получить субмикрозернистую рекристаллизованную структуру [20]. Наибольшее влияние ДР проявляется при температурах 100 и $150{ }^{\circ} \mathrm{C}$, т.е. когда термоактивированные зародыши отсутствуют, или их количество невелико. После деформации сдвигом под давлением доля «чистых» рекристаллизованных зерен больше, чем после РКУП (рис. 5). Например, после нагрева при $100{ }^{\circ} \mathrm{C}$ в течение 10 ч в образцах, деформированных сдвигом под давлением, остается около $10 \%$ нерекристаллизованной структуры, а в образце, деформированном РКУП, рекристаллизованные зерна составляют только 50 \% структуры. На рис. 5 видно, что скорость рекристаллизации наибольшая в структуре, претерпевшей полную ДР. На рис. 6 приведена структура меди после деформации и отжига при $100{ }^{\circ} \mathrm{C}$. Ячеистая и субзеренная структуры, сформировавшиеся при деформации, остаются без изменения (рис. 6 б, в). Происходит рост отдельных зерен, присутствующих в структуре до отжига. В ДР-структуре также наблюдается опережающий рост некоторых зерен, приводящий к значительной разнозернистости (рис. 6 a). Средний размер крупного рекристаллизованного зерна достигает 15-20 мкм, размеры элементов структуры нерекристаллизованной матрицы остаются неизменными.
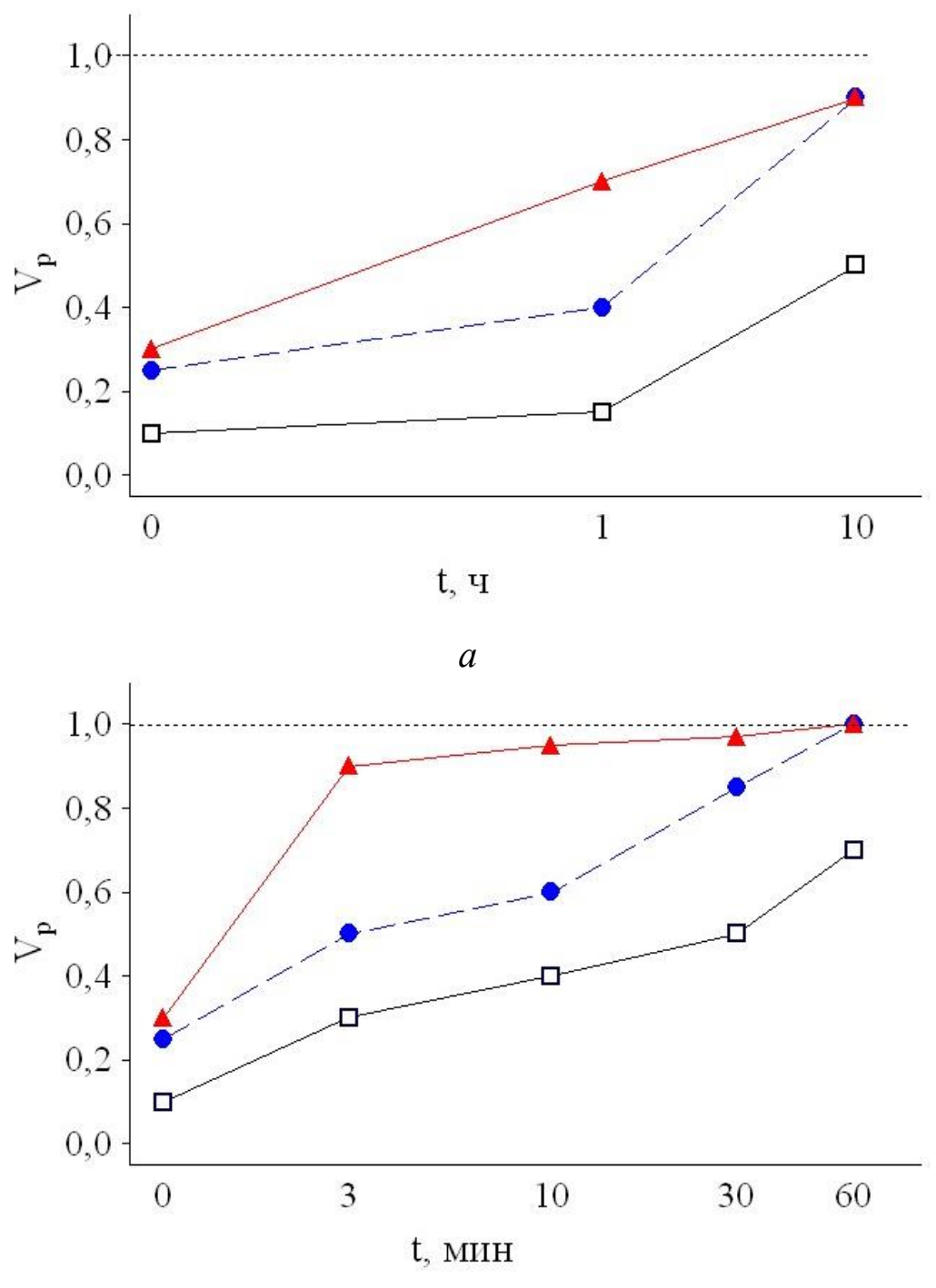

$\sigma$

Рис. 5. Доля рекристаллизованной структуры после деформации и отжига:

$a-100{ }^{\circ} \mathrm{C} ; \sigma-150{ }^{\circ} \mathrm{C}$. РКУП (口) и сдвиг под давлением с поворотом наковальни на $45^{\circ}(\bullet)$ и 5 оборотов $(\boldsymbol{\Delta})$ 
Рекристаллизация при $150{ }^{\circ} \mathrm{C}$ в образцах, деформированных сдвигом на 5 оборотов, практически завершается за 30 мин (рис. 5 б), а после деформации на $45^{\circ}$ - за 1 ч. Наибольший размер статически рекристаллизованного зерна наблюдается в исходно динамически рекристаллизованной структуре (рис. 3 б). Нагрев в течение 1 ч при этой температуре не приводит к окончанию рекристаллизации в образцах, деформированных РКУП (рис. 56 и 7 a). Таким образом, ДР приводит к формированию при низкотемпературной рекристаллизации крупнозернистой структуры, характеризующейся сильной размерной неоднородностью (рис. 7).

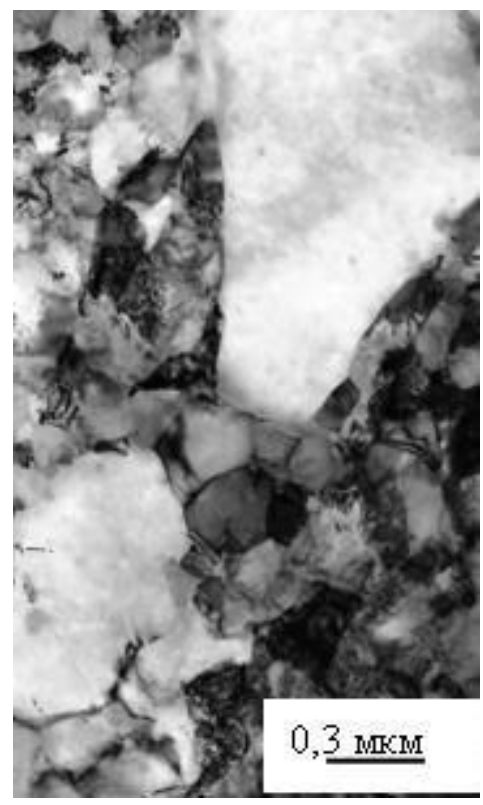

$a$

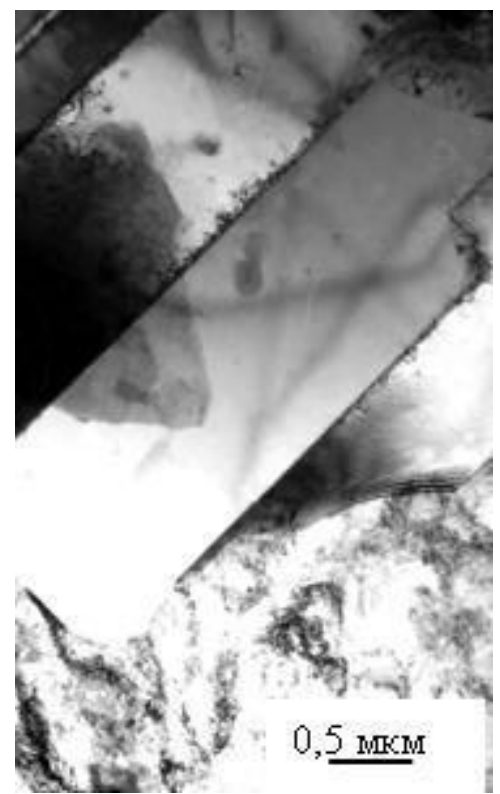

б

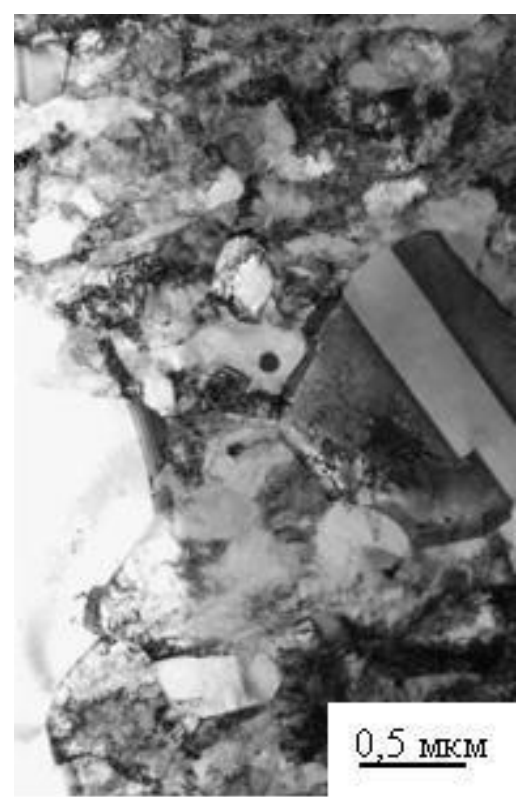

B

Рис. 6 Микроструктура меди после деформации и отжига при $100^{\circ} \mathrm{C}: a-5$ оборотов, отжиг в течение 1 ч; $\sigma-45^{\circ}$ отжиг в течение 10 ч; $в$ - РКУП, отжиг в течение 10 ч

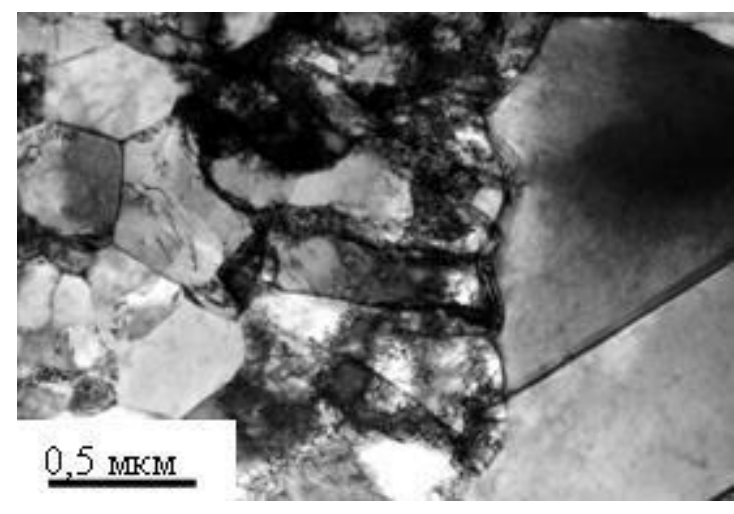

$a$

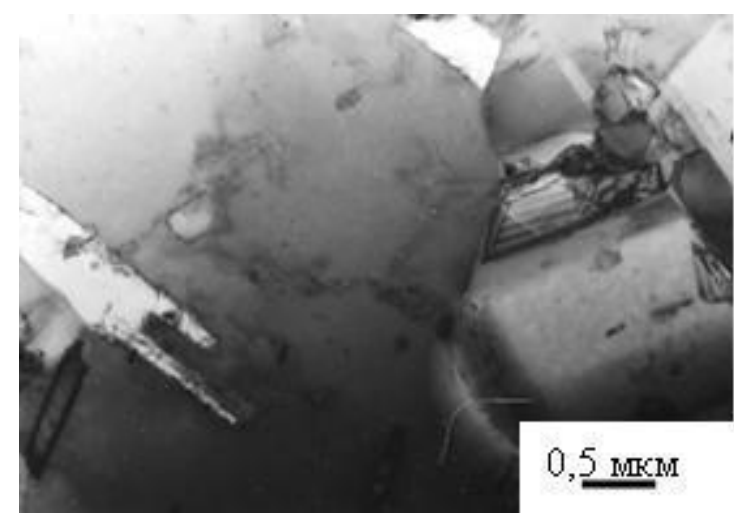

6

Рис. 7 Микроструктура меди после деформации и отжига при $150^{\circ} \mathrm{C}, 1 ч:$ $a$ - РКУП; $\sigma-5$ оборотов

Повышение температуры нагрева до $200{ }^{\circ} \mathrm{C}$ приводит к завершению рекристаллизации после выдержки 30 мин независимо от величины и способа деформации (рис. 8). Вид кинетических зависимостей изменяется (рис. 4). На них появляется перегиб, соответствующий завершению первичной рекристаллизации. С увеличением времени выдержки скорость 
роста зерна уменьшается. Такой же характер кинетических зависимостей наблюдали при низкотемпературной рекристаллизации чистого железа в работе [19].

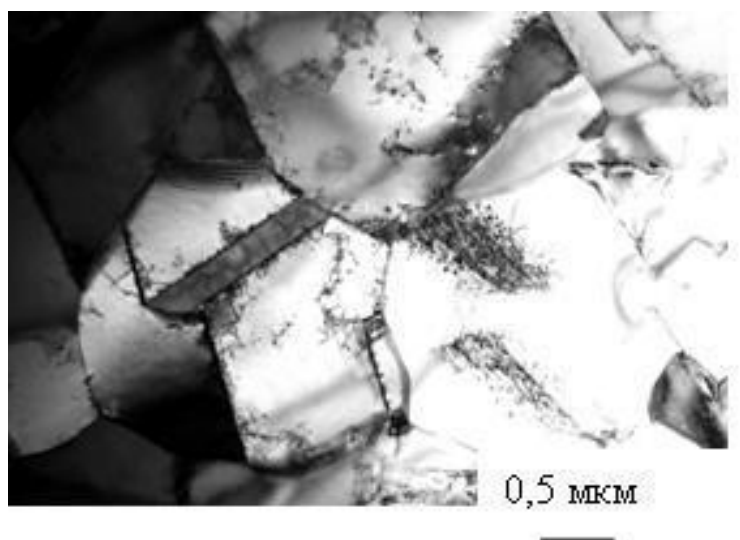

Рис. 8 Микроструктура меди после деформации РКУП и отжига при 200 C, 30 мин

В таблице представлены значения среднего размера элементов структуры от температуры отжига продолжительностью 1 ч.

Средний размер элементов структуры меди после деформации разными методами и отжига в течение 1 ч

\begin{tabular}{|c|c|c|c|}
\hline \multirow{2}{*}{ Температура отжига, ${ }^{\circ} \mathrm{C}$} & \multicolumn{3}{|c|}{ Метод деформации } \\
\cline { 2 - 4 } & \multicolumn{2}{|c|}{ Сдвиг под давлением } & \multirow{2}{*}{ РКУП } \\
\cline { 2 - 4 } & $45^{\circ}$ & 5 об. & 0,5 \\
\hline До отжига & 0,3 & 0,2 & 3,6 \\
\hline 100 & 2,0 & 15 & 10 \\
\hline 150 & 7,6 & 19 & 11 \\
\hline 200 & 7,1 & 13 & 8,2 \\
\hline 300 & 8,0 & 9,0 & 11 \\
\hline 400 & 13 & 9,6 & \\
\hline
\end{tabular}

В соответствии с рис. 5 после деформации сдвигом под давлением на 5 оборотов наковальни при всех температурах отжига эти элементы структуры представляют собой рекристаллизованные зерна. В образцах, деформированных на $45^{\circ}$, структура полностью состоит из рекристаллизованных зерен после отжига при $150{ }^{\circ} \mathrm{C}$ и выше, а в образцах, деформированных РКУП, - после отжига при $200{ }^{\circ} \mathrm{C}$ и выше. Видно, что в полностью динамически рекристаллизованном материале (5 об.) отжиг при температуре выше $150{ }^{\circ} \mathrm{C}$ приводит к уменьшению среднего размера зерна. Это, вероятно, связано с повышением плотности центров рекристаллизации, благодаря формированию термоактивированных центров в дополнение к созданным при деформации. Средний размер зерна в образцах с частично динамически рекристаллизованной структурой $\left(45^{\circ}\right.$, РКУП) меняется мало. Это можно объяснить малым числом центров рекристаллизации, созданных при деформации. Следует отметить, что при повышении температуры отжига выше $200{ }^{\circ} \mathrm{C}$, т.е. выше температуры термоактивированного зарождения, влияние ДР на размер статически рекристаллизованного зерна уменьшается. После отжига при 300-400 ${ }^{\circ} \mathrm{C}$ независимо от предшествующей обработки формируется структура с размером зерна около 10 мкм, при этом размерная неоднородность структуры сохраняется (рис. 9). 


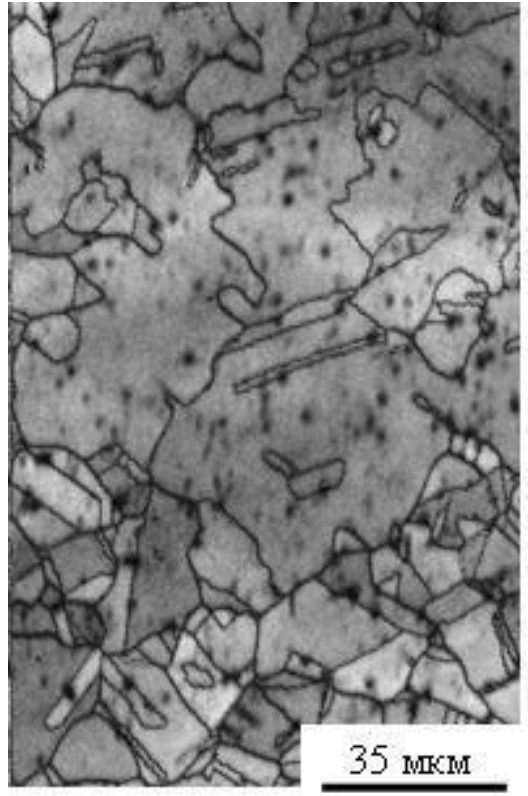

$a$

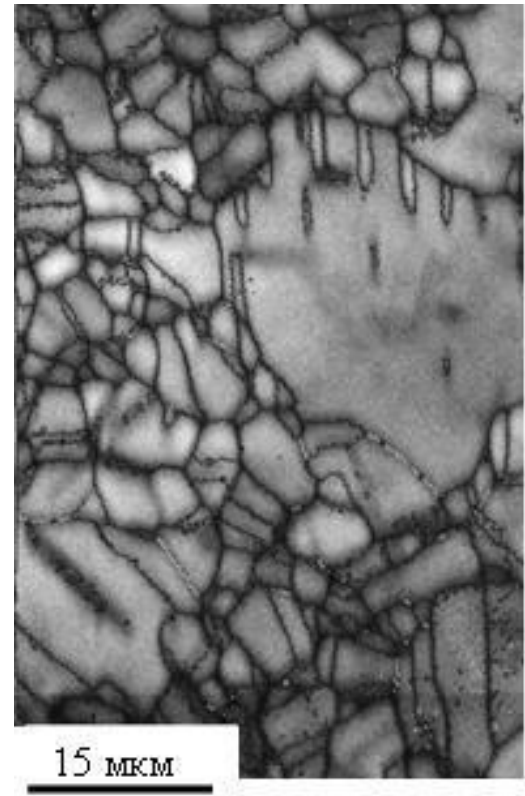

$\sigma$

Рис. 9. Микроструктура меди после деформации сдвигом под давлением и отжига при $400{ }^{\circ} \mathrm{C}, 1$ ч: $a-45^{\circ} ; \sigma-5$ оборотов

\section{4. Заключение}

В настоящей работе исследовано влияние динамической рекристаллизации, протекающей в процессе деформации, на изменение структуры при последующем нагреве меди марки Моб.

Показано, что варьирование условий деформации меди обеспечивает различную степень развития динамической рекристаллизации, динамического возврата и деформационного упрочнения. Развитие рекристаллизации в процессе деформации (динамической рекристаллизации) приводит к формированию наиболее крупнозернистой и размерно неоднородной структуры при последующей низкотемпературной статической рекристаллизации.

Повышение температуры нагрева в интервале 150-400 $\mathrm{C}$ (выше температуры термоактивированного зарождения) приводит к уменьшению среднего размера зерна в полностью динамически рекристаллизованном материале и мало меняет размер зерна в образцах с частично динамически рекристаллизованной структурой.

По завершении первичной рекристаллизации в меди не удается получить субмикрозернистую рекристаллизованную структуру. Наиболее мелкозернистая структура со средним размером зерна 4-7 мкм формируется после кратковременного отжига при $300{ }^{\circ} \mathrm{C}$.

\section{Благодарность}

Работа выполнена в рамках государственного задания ФАНО России (тема «Кристалл» №01201463333) при частичной поддержке проекта УрО РАН №15-17-2-11. Электронно-микроскопическое исследование выполнено в ЦКП «Испытательный центр нанотехнологий и перспективных материалов» ИФМ УрО РАН.

\section{Литература}

1. Горелик С. С., Добаткин С. В., Капуткина Л. М. Рекристаллизация металлов и сплавов. - М. : МИСИС, 2005. - 432 с.

2. Voronova L. M., Degtyarev M. V., Chashchukhina T. I. Recrystallization of the ultradispersed structure of pure iron formed at different stages of the deformation-induced 
strain hardening // Physics of Metals and Metallography. - 2007. - Vol. 104, iss. 3. - P. 262-273. DOI: 10.1134/S0031918X07090086.

3. Effect of Annealing Temperature on the Recrystallization of Nickel with Different Ultradisperse Structures / Yu. G. Krasnoperova, M. V. Degtyarev, L. M. Voronova, T. I. Chashchukhina // Physics of Metals and Metallography. - 2016. - Vol. 117, no. 3. - P. 267-274. - DOI: 10.1134/S0031918X16030078.

4. Peculiarities of low-temperature recrystallization of nickel and copper / N. A. Smirnova, V. I. Levit, V. P. Pilyugin, R. I. Kuznetsov, M. V. Degtyarev // Fizika Metallov i Metallovedenie. 1986. - Vol. 62, iss. 3. - P. 566-570.

5. Correlation between the copper structure and temperature-rate parameters of pressureinduced shear deformation / M. V. Degtyarev, T. I. Chashchukhina, M. Yu. Romanova, L. M. Voronova // Doklady Physics. - 2004. - Vol. 49, no. 7. - P. 415-418. - DOI: $10.1134 / 1.1784855$.

6. Influence of the relaxation processes on the structure formation in pure metals and alloys under high-pressure deformation / M. V. Degtyarev, T. I. Chashchukhina, L. M. Voronova, A. M. Patselov, V. P. Pilyugin // Acta Materialia. - 2007. - Vol. 55, iss. 18. - P. 6039-6050. - DOI: 10.1016/j.actamat.2007.04.017.

7. Low-Temperature Recrystallization of Copper Rolled at 77 and $20 \mathrm{~K} / \mathrm{I}$. A. Gindin, B. G. Lazarev, J. D. Starodub, M. B. Lazareva // Dokl. Akad. Nauk SSSR. - 1966. - Vol. 171. No. 3. - P. 552-554.

8. Fragmentation and dynamic recrystallization of copper at large and very large plastic deformations / V. M. Bykov, V. A. Likhachev, Yu. A. Nikonov, L. L. Serbina, L. I. Shibalova // Fizika Metallov i Metallovedenie. - 1978. - Vol. 45, no. 1. - P.163-169.

9. Anomalous grain growth in nano- and microcrystalline metals produced by equal-channel angular pressing methods. Part I. Structural studies / V. N. Chuvil'deev, V. I. Kopylov, A. V. Nokhrin, I. M. Makarov, L. M. Malashenko, V. A. Kukareko // Materialovedenie. - No. 4. P. 9-17.

10. Кристаллографический анализ субмикрокристаллической структуры, полученной РКУ прессованием высокочистой меди / В. И. Копылов, И. М. Макаров, Е. В. Нестерова, В. В. Рыбин // Вопросы материаловедения. - 2002. - № 1 (29). - С. 273-278.

11. Amirkhanov N. M., Islamgaliev R. K., Valiev R. Z. Thermal relaxation and grain growth upon isothermal annealing of ultrafine-grained copper produced by severe plastic deformation // Fizika Metallov i Metallovedenie. - 1998. - Vol. 86, iss. 3. - P. 99-105.

12. Effect of impurities on dynamic recrystallization in copper deformed in bridgman anvils / D. K. Orlova, T. I. Chashchukhina, L. M. Voronova, M. V. Degtyarev, Yu. G. Krasnoperova // Diagnostics, Resource and Mechanics of materials and structures. - 2015. - Iss. 5. - P. 90-98. DOI: 10.17804/2410-9908.2015.5.090-098. - $\quad$ URL: http://dream- journal.org/DREAM_Issue_5_2015_Orlova_D.K._et_al._090_098.pdf (accessed 13.09.2016).

13. Shirinkina I. G., Brodova I. G., Astafiev V. V. Thermal stability of the ultrafine amts aluminum alloy after high strain-rate deformation // Diagnostics, Resource and Mechanics of materials and structures. - 2015. - Iss. 5. - P. 72-79. - DOI: 10.17804/2410-9908.2015.5.072-079.

- URL: http://dream-journal.org/DREAM_Issue_5_2015_Shirinkina_I.G._et_al._072_079.pdf (accessed 13.09.2016).

14. Гусев А. И. Нанокристаллические материалы: методы получения и свойства. - Екатеринбург : УрО РАН, 1998. - 200 с.

15. Пилюгин В. П. Структурные и фазовые превращения в сплавах железа при деформации под высоким давлением : дис. ... канд. физ.-мат. наук : 01.04.07. - Екатеринбург, 1993. $200 \mathrm{c}$.

16. Chashchukhina T. I., Degtyarev M. V., Voronova L. M. Effect of Pressure on the Evolution of Copper Microstructure upon Large Plastic Deformation // Physics of Metals and Metallography.

- 2010. - Vol. 109, iss. 2. - P. 201-209. - DOI: 10.1134/S0031918X10020122. 
17. Процессы пластического структурообразования металлов / В. М. Сегал, В. И. Резников, В. И. Копылов, Л. А. Павлик, В. Ф. Малышев. - Минск : Навука і тэхніка, 1994. $-232 \mathrm{c}$.

18. Установление соответствия между степенью деформации, твердостью и размерами элементов структуры железа и конструкционных сталей при большой пластической деформации различными способами. / М. В. Дегтярев, Т. И. Чащухина, Л. М. Воронова, В. И. Копылов // Физическая мезомеханика. - 2013. - Т. 16, № 6. - С. 71-80.

19. Low-temperature recrystallization of pure iron deformed by shear under pressure / M. V. Degtyarev, L. M. Voronova, T. I. Chashchukhina // Physics of Metals and Metallography. 2004. - Vol. 97, no. 1. - P. 72-81.

20. Influence of aging and strain on the structure and mechanical properties of chromiumzirconium bronze / N. Yu. Frolova, V. I. Zeldovich, I. V. Khomskaya, A. E. Kheifets, E. V. Shorokhov // Diagnostics, Resource and Mechanics of materials and structures. - 2015. Iss. 5. - P. 99-108. - DOI: 10.17804/2410-9908.2015.5.099-108 . - URL: http://dreamjournal.org/DREAM_Issue_5_2015_Frolova_N._Yu._et_al._099_108.pdf (accessed 13.09.2016). 